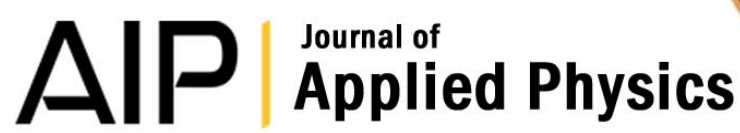

Origin of ferromagnetism and oxygen-vacancy ordering induced crosscontrolled magnetoelectric effects at room temperature

X. K. Wei, T. Zou, F. Wang, Q. H. Zhang, Y. Sun et al.

Citation: J. Appl. Phys. 111, 073904 (2012); doi: 10.1063/1.3696979

View online: http://dx.doi.org/10.1063/1.3696979

View Table of Contents: http://jap.aip.org/resource/1/JAPIAU/v111/i7

Published by the American Institute of Physics.

\section{Additional information on J. Appl. Phys.}

Journal Homepage: http://jap.aip.org/

Journal Information: http://jap.aip.org/about/about_the_journal

Top downloads: http://jap.aip.org/features/most_downloaded

Information for Authors: http://jap.aip.org/authors

\section{ADVERTISEMENT}

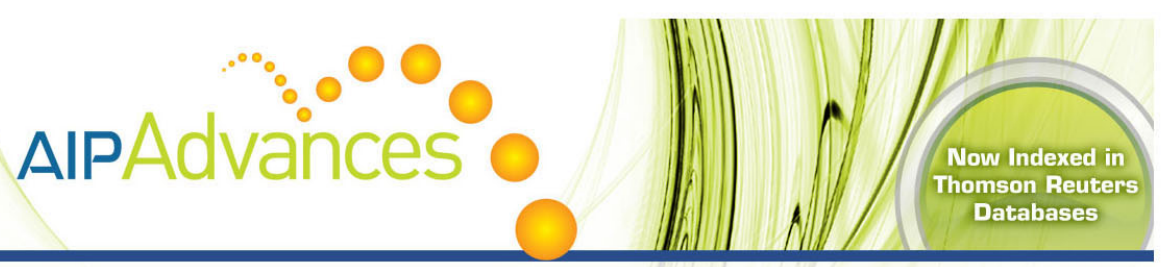

\section{Explore AIP's open access journal: Rapid publication \\ - Article-level metrics \\ - Post-publication rating and commenting}




\title{
Origin of ferromagnetism and oxygen-vacancy ordering induced cross-controlled magnetoelectric effects at room temperature
}

\author{
X. K. Wei, ${ }^{1}$ T. Zou, ${ }^{1}$ F. Wang, ${ }^{1}$ Q. H. Zhang, ${ }^{1}$ Y. Sun, ${ }^{1}$ L. Gu, ${ }^{1,2}$ A. Hirata, ${ }^{2}$ M. W. Chen, ${ }^{2}$ \\ Y. Yao, ${ }^{1}$ C. Q. Jin, ${ }^{1}$ and R. C. Yu ${ }^{1, a)}$ \\ ${ }^{1}$ Beijing National Laboratory for Condensed Matter Physics, Institute of Physics, \\ Chinese Academy of Sciences, Beijing 100190, China \\ ${ }^{2}$ WPI Advanced Institute for Materials Research, Tohoku University, Sendai 980-8577, Japan
}

(Received 3 November 2011; accepted 21 February 2012; published online 6 April 2012)

In dilute magnetic oxide hexagonal $\mathrm{Ba}\left(\mathrm{Ti}_{0.9} \mathrm{Fe}_{0.1}\right) \mathrm{O}_{2.81}$ bulk ceramic, we report on combined ferromagnetism and improper ferroelectricity as well as cross-controlled magnetoelectric effects at room temperature. The annular-bright-field $(\mathrm{ABF})$ imaging technique in scanning transmission electron microscopy (STEM) demonstrates an oxygen vacancy ordering in the hexagonal closestpacked Ba1-O1 layers and severe distortion of the octahedra and pyramids. Strong dependencies of the susceptibility on temperature and magnetic field as well as the frequency dependence of magnetization under an $a c$ electric field reveal that the intrinsic ferromagnetism of the highly insulating system dynamically evolves from a paramagnetic ground state, and dynamic exchanges of trapped electrons in the ordered polarons are attributed to the ferromagnetic interaction. Accordingly, aided by the motion of oxygen vacancies, responses of the trapped electrons to the $a c$ magnetic field result in the reversal of magnetically induced voltages between high and low states. Our results not only expand our understanding on the magnetoelectric coupling mechanism, but also provide a grand opportunity toward designing novel multiferroic materials through introducing ordered point defects into a centrosymmetric matrix. (C) 2012 American Institute of Physics. [http://dx.doi.org/10.1063/1.3696979]

\section{INTRODUCTION}

The coexistence of two or more physical degrees of freedoms in a material, which are intricately coupled and delicately balanced, provides a variety of unprecedented phenomena. The magnetoelectric (ME) effects arising from the interplay between magnetic and ferroelectric orders in multiferroic materials are considered to be an important issue that holds promises in designing novel logic devices. ${ }^{1}$ Among the known multiferroics, apart from the universal low ME coupling temperature, the excessive electrical conduction ${ }^{2}$ and the high operating magnetic field $(H)$, for example, in magnetic ferroelectrics hexagonal $R \mathrm{MnO}_{3}$ and orthorhombic $R \mathrm{Mn}_{2} \mathrm{O}_{5}$ $(R=$ rare earths $),{ }^{3,4}$ as well as the rare cross-control of polarization $(P)$ by $H$ and magnetization $(M)$ by electric field $(E)$ at room temperature has severely hampered their practical applications. Hence, searching for a new mechanism is crucial to resolving the aforesaid bottleneck problems.

Historically, the $d^{0}$-ness criteria for ferroelectricity (FE) generally denies the coexistence of ferromagnetism (FM) and FE in magnetic $d^{\mathrm{n}}$-ions doped perovskite oxides owing to the Hund's rule. ${ }^{5,6}$ Nevertheless, this criteria is invalid for the hexagonal perovskites, such as $R \mathrm{MnO}_{3}(R=\mathrm{Ho}-\mathrm{Lu}$, Y). ${ }^{3,5}$ Recently, triggered by the design of novel ME multiferroic materials at room temperature, ${ }^{7-9}$ trivalent Fe-doped $6 \mathrm{H}-\mathrm{BaTiO}_{3}$ has received much attention because of their unusual high-temperature $\mathrm{FM},{ }^{10-13}$ which involves with the appearance of oxygen vacancies (Vos) at O1-sites. ${ }^{11-17}$

\footnotetext{
a) Author to whom correspondence should be addressed. Electronic mail: rcyu@aphy.iphy.ac.cn.
}

However, origin of the intrinsic FM in the hexagonal polymorph has not been clarified. According to the low-spin state of the doped $\mathrm{Fe}^{3+}$ ions at the M2 site revealed by the ab-initio calculation, ${ }^{10} \mathrm{FE}$ may possibly be compatible with the FM. However, neither has the coexistence of FE with FM been realized.

Systematic studies on both dilute $(\leq 10 \mathrm{~mol} \%)$ and dense $(>10 \mathrm{~mol} \%) \mathrm{Fe}$-doped hexagonal $\mathrm{BaTiO}_{3}$ have revealed that the FM at room temperature can be mediated by both the vacancy content of oxygen ${ }^{13,16}$ and the doping concentration of Fe ions. ${ }^{11,18}$ Meanwhile, the concentration of Vos plays a crucial role in both the magnetic and the electrical properties of the doped and the prototypic hexagonal $\mathrm{BaTiO}_{3} \cdot{ }^{16,19,20} \mathrm{On}$ the one hand, the coordination of transition metals at the M2 site changes owing to the transformation from face-sharing octahedra to edge-sharing pyramids as one $\mathrm{O} 1$ atom becomes deficient among the nearest three or to corner-sharing tetrahedra as two $\mathrm{O} 1$ atoms are missed. ${ }^{14}$ On the other hand, the band structure varies because of the creation of impurity states within the bandgap ${ }^{21}$ and partial reduction of $\mathrm{Ti}^{4+}$ to $\mathrm{Ti}^{3+}$ due to the compensation of electrons to the vacancies. ${ }^{19}$ Usually Fe keeps the trivalent state as its substitution content for $\mathrm{Ti}$ is less than $67 \mathrm{~mol} \% .^{11,14}$ Therefore, considering its considerable doping concentration (from $\sim 1$ to $67 \mathrm{~mol} \%$ or even higher), ${ }^{10,15,22} \mathrm{Fe}$-doped hexagonal $\mathrm{BaTiO}_{3}$ is a system full of physical fundamentals because of its tunability in both crystal and electronic structures as well as its physical properties.

Here, we report the coexistence of FM and improper FE, and the cross-controlled magnetoelectric effects at room temperature induced by vacancy ordering of oxygen in electrically insulating hexagonal $\mathrm{Ba}\left(\mathrm{Ti}_{0.9} \mathrm{Fe}_{0.1}\right) \mathrm{O}_{2.81}$ (BTF10). 
Our convincing experimental results reveal that the intrinsic FM derives from a dynamic exchange interaction of trapped electrons in the ordered polarons. Our study not only verifies the previous prediction on how to combine the magnetic and ferroelectric orders in one compound, ${ }^{23}$ but also provides a new mechanism in designing multiferrocity.

\section{EXPERIMENTAL DETAILS}

The sample was prepared with the solid state reaction method using stoichiometric amounts of $\mathrm{BaCO}_{3}, \mathrm{TiO}_{2}$, and $\mathrm{Fe}_{2} \mathrm{O}_{3} .{ }^{24}$ The X-ray diffraction (XRD) experiments were carried out on a Philips X'Pert Pro powder diffractometer $\left(\mathrm{CuK} \alpha_{1}=1.5406 \AA\right)$. Transmission electron microscopy (TEM) observations were performed on Tecnai F20 and JEOL $2100 \mathrm{~F}$ equipped with double Cs-correctors. The absorption edges of Ti- $L_{23}$ are collected in image mode using the aperture with a diameter of $20 \mu \mathrm{m}$ at room temperature. The dielectric constant $\left(\varepsilon_{\mathrm{r}}\right)$ and loss tangent $(\tan \delta)$, polarization $(P)$ versus electric field $(E)$ loops and leakage current were measured on HP 4192 A and TF 2000, respectively. The magnetism measurements were performed on Quantum Design PPMS, in which both the zero-field-cooling (ZFC) and field-cooling (FC) curves are recorded in warming cycles. After poling the sample under a $d c E_{\text {pole }}=6 \mathrm{kV} / \mathrm{cm}$, the dynamic ME coefficient $\left(\alpha_{\mathrm{EM}}=\delta \mathrm{E} / \delta H\right)$ inspired by a 5 Oe $a c H$ was collected along the poling direction. ${ }^{25}$ Control of $M \times E$ was performed when a permanent $d c H=10 \mathrm{kOe}$ was applied perpendicular to the $a c$ field with amplitude of $E=0.8 \mathrm{kV} / \mathrm{cm}$. Silver paste was painted on sample surfaces as electrodes.

\section{RESULTS AND DISCUSSION}

\section{A. Structure investigations}

According to the Raman analysis carried out on polycrystalline $\mathrm{Ba}\left(\mathrm{Ti}_{1-\mathrm{x}} \mathrm{Fe}_{\mathrm{x}}\right) \mathrm{O}_{3-\delta}$, tetragonal and hexagonal phases always coexist in the doping range of $0.02 \leq \mathrm{x} \leq 0.12 .^{26}$ However, the temperature-composition phase diagram investigation on dilute $(\mathrm{x} \leq 0.1)$ doping reveals that higher sintering temperature can effectively purify the compound to be hexagonal. ${ }^{24,27}$ Meanwhile, our former studies on Fe-doped $\mathrm{BaTiO}_{3}$ ceramics also indicate that concentration of the hexagonal phase in the doped system can be influenced greatly by the purity of the raw materials. Although XRD is not as sensitive as Raman scattering ${ }^{26}$ in detecting the secondary phase, our systematic TEM observations on BTF10 reveal that a secondary phase exists but is quite rare. Figure 1 shows the room-temperature XRD pattern of BTF10 refined using non-centrosymmetric space group $P-62 c\left(R_{\mathrm{p}}=5.96 \%\right.$, $\left.R_{\mathrm{wp}}=8.20 \%, \chi^{2}=1.69\right)$. The refinements reveal that the doped sample has lattice parameters of $a=5.721 \AA$ and $c=13.990 \AA$, Vos appear at the O1-site only and the doped $\mathrm{Fe}^{3+}$ ions prefer to occupy the M2-site. ${ }^{14}$ Actually, this refinement result is obtained as a large part of the Til ions that are fixed at the trivalent state and $\mathrm{Ti} 2$ ions are fixed at the divalent state. This may not be the real case in the sample, but it strongly indicates the reduction of partial $\mathrm{Ti}^{4+}$ ions to lower valence states. Similar refinement results can also be obtained when the refinement is performed using centrosym-

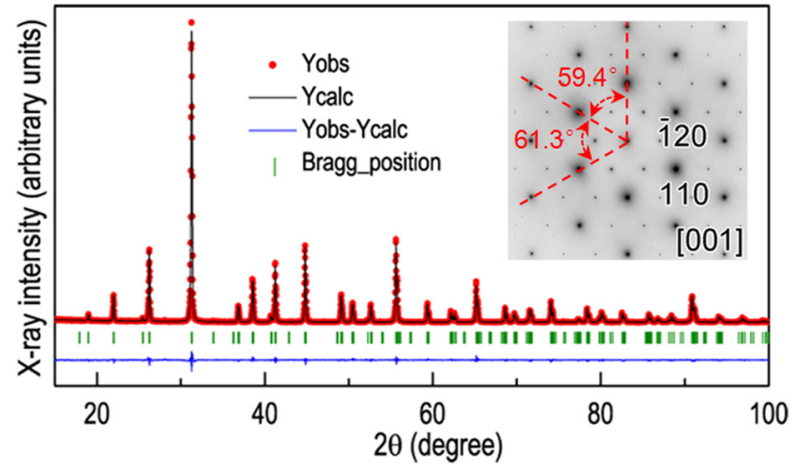

FIG. 1. The refined XRD pattern of BTF10 using space group $P-62 c$ at room temperature. The insert is the ED pattern taken along the [001] zone axis.

metric space group $\mathrm{P}_{3} / m m c$ of hexagonal $\mathrm{BaTiO}_{3}$. The successful substitution of $\mathrm{Fe}$ for $\mathrm{Ti}$ as well as the accompanied Vos can further be identified from distortion of the hexagonal matrix, as the electron diffraction (ED) shown in the insert of Fig. 1, which is evidenced by the deviation of angles between adjacent $\{110\}$ reflections from $60^{\circ}$.

To obtain the vacancy distribution of oxygen in the hexagonal matrix, we performed the annular-bright-field (ABF) image observation using a scanning transmission electron microscopy technique, which is reliable in imaging both light and heavy atoms. ${ }^{28}$ The lower right corner panel in Fig. 2(a) shows the ABF image collected along [010] direction with the refined structure inserted for comparison. The sharp image contrast between adjacent hexagonal $(h)$ closestpacked Ba1-O1 layers can clearly be seen from the colored image. From the intensity profiles shown in Fig. 2(b), which are correspondingly extracted from Fig. 2(a), we can clearly see that the contrast difference results from different $\mathrm{O} 1$ atom occupation in the hexagonal layers. This demonstrates the presence of vacancy ordering at the $\mathrm{O} 1$ site in BTF10. However, the intensity profile extracted from the $h$ ' layer suggests that there are still oxygen atoms occupying the $\mathrm{O} 1$ site which is located between adjacent Ba1/O1 columns, merely that they deviate from the atomic site of the refined structure. Therefore, we can generally conclude that the vacancy ordering is caused by the transformation from face-sharing octahedra to edge-sharing pyramids in this hexagonal matrix. The schematic structure obtained from refinement is shown in Figs. 2(d) and 2(e). Here, configuration of the edge-sharing pyramids is scaled by the introduced dihedral angle $\varphi$, bridging angle $\theta$, and rotation angle $\phi$. Actually, the coordination of M2 cations related with the $h$ ' layer needs to be further determined, which will be stressed later.

Similarly, clear image contrast between cubic (c) closest-packed Ba2-O2 layers can also be identified from the colored $\mathrm{ABF}$ image. For the $c$ layers, the $\mathrm{O} 2$ atoms between adjacent $\mathrm{Ba} 2 / \mathrm{O} 2$ columns only occupy one kind of crystallographic site, whereas for the c' layers, $\mathrm{O} 2$ atoms take up two kinds of atomic sites (see Fig. 2(a)). This reveals that associated with the vacancy ordering, the octahedral and pyramidal cages are severely distorted. Correspondingly, superstructure reflection is thus not observed in the ED pattern (Fig. 2(f)). If we consider the compensation of electrons to the Vos, $\mathrm{Ti}^{4+}$ 
(a) $\frac{B a_{1}}{O_{1}} O_{1} \frac{B a_{1}}{O_{1}} O_{1} \frac{B a_{1}}{O_{1}} O_{1} \frac{B a_{1}}{O_{1}} O_{1} \frac{B a_{1}}{O_{1}} O_{1} \frac{B a_{1}}{O_{1}} O_{1}$

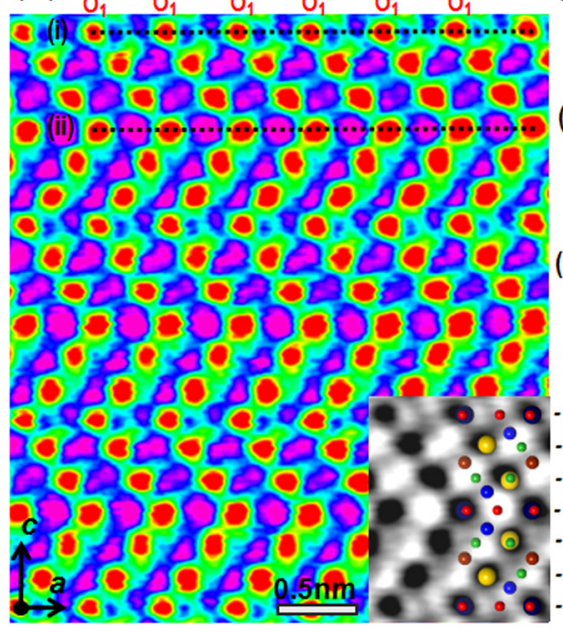

(b) $O_{1} \frac{B a_{1}}{O_{1}} O_{1} \frac{B a_{1}}{O_{1}} O_{1} \frac{B a_{1}}{O_{1}}, \frac{B a_{1}}{O_{1}}, \frac{B a_{1}}{O_{1}} O_{1} \frac{B a_{1}}{O_{1}}$

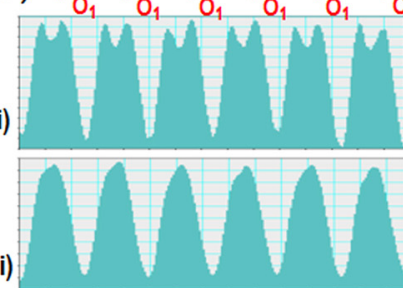

$\begin{array}{llllllll}0.0 & 0.4 & 0.8 & 1.2 & 1.6 & 2.0 & 2.4 & 2.8\end{array}$ Intensity (a.u.) 0 h-Ba101 c'- $\mathrm{Ba2} 22_{3}$. $-c^{\prime}-\mathrm{Ba}_{2} \mathrm{O} 2_{3}$. h'-Ba1012' c. $\mathrm{Ba} 2 \mathrm{O} 2_{3}$. c- $\mathrm{Ba} 2 \mathrm{O} 2_{3}-$ - $h-\mathrm{Ba}_{101_{3}}$ $\mathrm{nm}$ (c) (d)

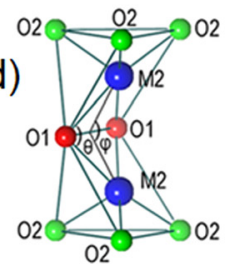

(e)

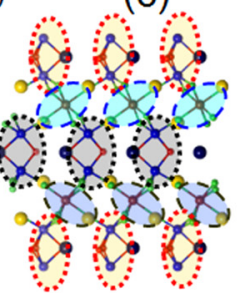

(f)
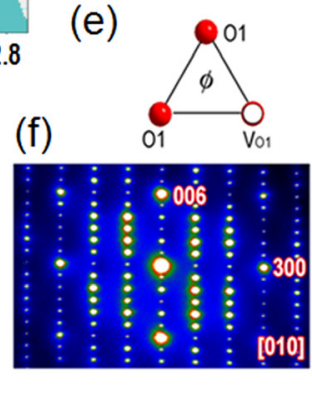

FIG. 2. (a) The colored ABF image of BTF10 taken along the [010] zone axis, red for $\mathrm{Ba}$ and pink for $\mathrm{O}$ atoms. The lower-right panel shows the original image and the unit cell obtained from refinements. The brown and blue atoms stand for atoms at M1 and M2 site, respectively. (b) The line-scan intensity profiles extracted from (a). (c) The polyhedral distortion and the schematic polaron ordering. $(\mathrm{d}, \mathrm{e})$ Parameters of dihedral angle $\varphi \approx$ $111.9^{\circ}$ for edge-sharing $\mathrm{O1}_{2} \mathrm{O}_{2}$ square planes and bridging angle $\theta \approx 113^{\circ}$ for M2-O1-M2 chain, rotation angle $\phi=2 \pi / 3$ for one $\mathrm{O} 1$ vacancy among the nearest three in the edge-sharing pyramids, respectively. The schematic configuration and values are obtained from XRD refinement. (f) The ED pattern taken along the [010] zone axis. ions at the M2 site may reduce to $\mathrm{Ti}^{3+}$ for those with pyramidal coordination. Subsequently, the distribution of electrons within the strongly anisotropic structure may possibly dimerize the edge-sharing pyramids, the face-sharing octahedra, as well as the M1-centered octahedra (bond length: M2-M2 = $2.729 \AA$, M1-M2 = $3.932 \AA)$. Therefore, the doped hexagonal matrix forms ordered arrangement of small polarons (see Fig. 2(c)).

\section{B. Magnetic and electrical properties}

Figure 3(a) shows the temperature dependence of inverse susceptibility $(1 / \chi)$ measured in $\mathrm{ZFC}$ and FC modes, respectively. Because of the structural anisotropy induced by vacancy ordering, the $\mathrm{ZFC}$ and FC curves strongly diverge over a wide temperature range; furthermore, their intersection at $T_{\mathrm{I}}$ increases from $255.6 \mathrm{~K}$ to $283 \mathrm{~K}$ as $H$ enhances from $1 \mathrm{kOe}$ to $2 \mathrm{kOe}$. Associated with the temperature dependence of the derivative of specific heat over temperature $(C / T)$, the isothermal $M$ reveals a transition from paramagnetism (PM) to FM at $T_{\mathrm{P}-\mathrm{F}}=25.6 \mathrm{~K}$ and an enhancement of the coercive field $\left(H_{\mathrm{C}}\right)$ with increasing temperature (see Fig. 3(b)). Comparatively, these behaviors are contrary to those in multiferroic bulks, such as $R_{0.5} A_{0.5} \mathrm{MnO}_{3}(R=\mathrm{Pr}$, $\mathrm{Nd}, \mathrm{Sm} ; A=\mathrm{Sr}, \mathrm{Ca}), \mathrm{LuFe}_{2} \mathrm{O}_{4}$, and $\mathrm{BiFeO}_{3} \cdot{ }^{29-31} \mathrm{With}$ respect to the positive role of $H$ to $T_{\mathrm{I}}$, similar behavior observed in epitaxial $\mathrm{La}_{0.7} \mathrm{Ca}_{0.3} \mathrm{MnO}_{3}$ and $\operatorname{Pr}_{0.7}\left(\mathrm{Sr}_{1-\mathrm{x}} \mathrm{Ca}_{\mathrm{x}}\right)_{0.3} \mathrm{MnO}_{3}$ thin films suggests that this may attribute to an exchange interaction of self-localized electrons in the small polarons. ${ }^{32}$

As is known, the deviation from Curie law is usually caused by zero-field splitting, ${ }^{33}$ which can lead to weak FM or canted antiferromagnetic ordering. Thus, the vacancy ordering and the polyhedral distortions must have played a vital role in generating the FM. Referring to the susceptibility

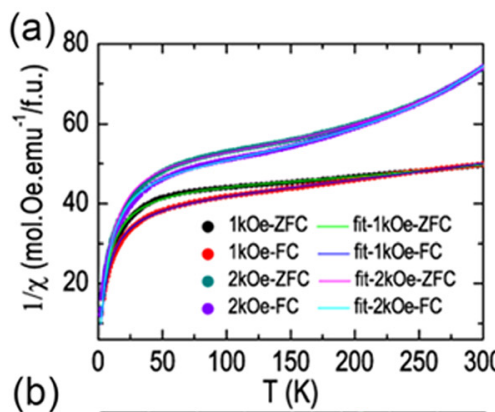

(c)

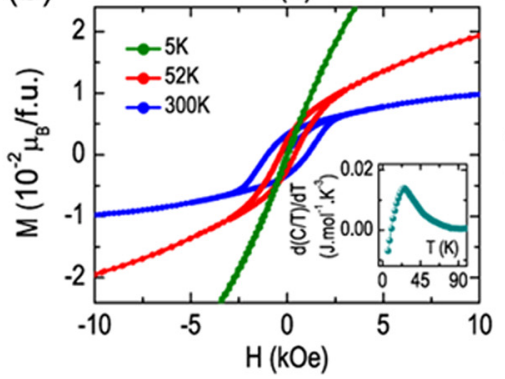

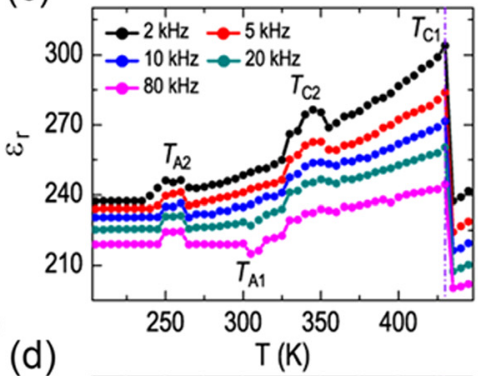

(d)

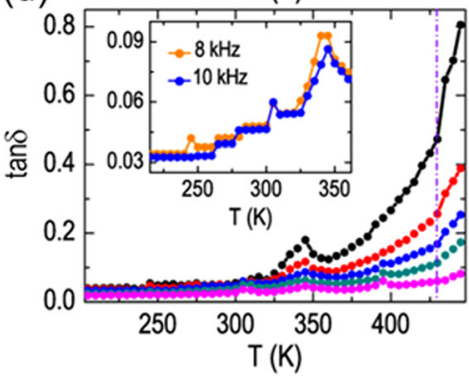

(e)

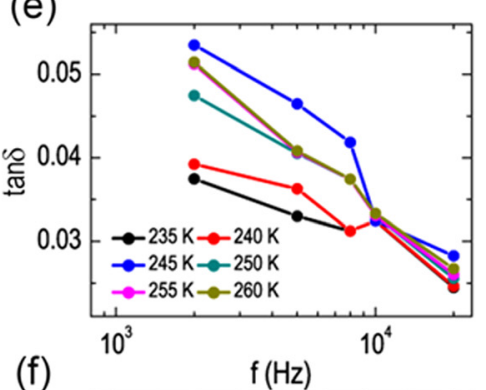

(f)

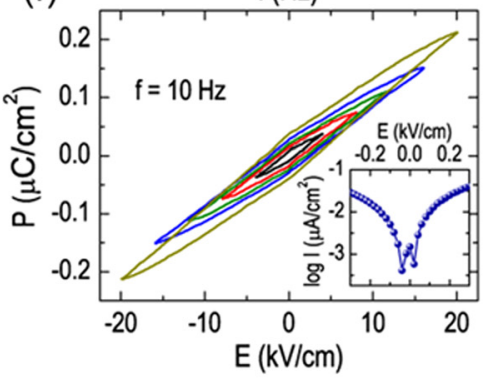

FIG. 3. (a) Temperature dependence of ZFC and FC $1 / \chi$ measured at 1 and $2 \mathrm{kOe}$, respectively. Solid lines are the fitted curves. (b) The magnetic hysteresis measured at 5,52 , and $300 \mathrm{~K}$, respectively. The insert is the temperature dependent $d(C / T) / d T$. (c, d) Temperature dependence of $\varepsilon_{\mathrm{r}}$ and tan $\delta$. The insert in $(\mathrm{d})$ is a magnified view of $\tan \delta$ measured at 8 and $10 \mathrm{kHz}$. (e) The frequency profile of $\tan \delta$ ranging from 235 to $260 \mathrm{~K}$. (f) Room-temperature ferroelectric loops measured at $f=10 \mathrm{~Hz}$. The insert is the leakage-current curve measured at room temperature. 
TABLE I. The fitted parameters for the susceptibility curves using expression (1). ${ }^{\mathrm{a}}$

\begin{tabular}{lccccc}
\hline \hline$H / \mathrm{kOe}$ & Mode & $p_{1}(H)$ & $p_{2}(H) / 10^{-2}$ & $p_{3}(H) / 10^{-6}$ & $p_{4}(H) / 10^{-8}$ \\
\hline 1 & ZFC & $0.148(2)$ & $2.13(1)$ & $1.6(7)$ & $-2.5(2)$ \\
1 & FC & $0.152(3)$ & $2.40(1)$ & $-14.8(8)$ & $-5.8(2)$ \\
2 & ZFC & $0.154(1)$ & $1.75(1)$ & $6.3(3)$ & $-7.1(1)$ \\
2 & FC & $0.149(2)$ & $1.89(1)$ & $-0.9(4)$ & $-6.3(1)$ \\
\hline \hline
\end{tabular}

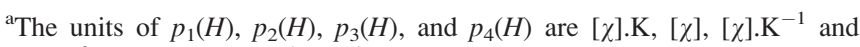

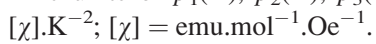

behaviors of low-dimensional organic binuclear complexes, ${ }^{34,35}$ in which the transition metals are fivefold coordinated and linked by a common edge, we find that a cut-off power series other than the Van Vleck equation ${ }^{33}$ can be used to explore the magnetism origin,

$$
\chi(H, T)=p_{1}(H) \cdot T^{-1}+p_{2}(H)+p_{3}(H) \cdot T+p_{4}(H) \cdot T^{2} .
$$

The strong dependencies of $\chi$ on $T$ and $H$ reveal that the FM in BTF10 derives from a dynamic phase transition and more details can be identified from the fitted parameters shown in Table I. The weakly $H$ dependent $p_{1}(H)$ actually is the Curie constant and dominates the ground-state PM. The constant $p_{2}(H)$ implies the net susceptibility, but it shows a strong dependence on $H$. Evidently, the linear item $p_{3}(H)$ attribute to the divergence of the ZFC and FC susceptibility curves and the quadratic $p_{4}(H)$ is another critical item in describing the exchange interaction of trapped electrons among the small polarons. ${ }^{36}$ Calculations on $p_{1}(H)$ yield that $(27.2 \pm 0.6) \mathrm{mol}$ $\% \mathrm{Ti}^{4+}$ ions are reduced to $\mathrm{Ti}^{3+}$ ions provided that all $\mathrm{Fe}^{3+}$ ions occupy the M2 site have a low spin state, ${ }^{10}$ and the vacancy content is determined to be of $\delta=0.186 \pm 0.003$ according to the electroneutrality. Correspondingly, the valency change of $\mathrm{Ti}$ ions is verified by our EELS study at room temperature. Figure 4(a) shows the Ti- $L_{23}$ absorption

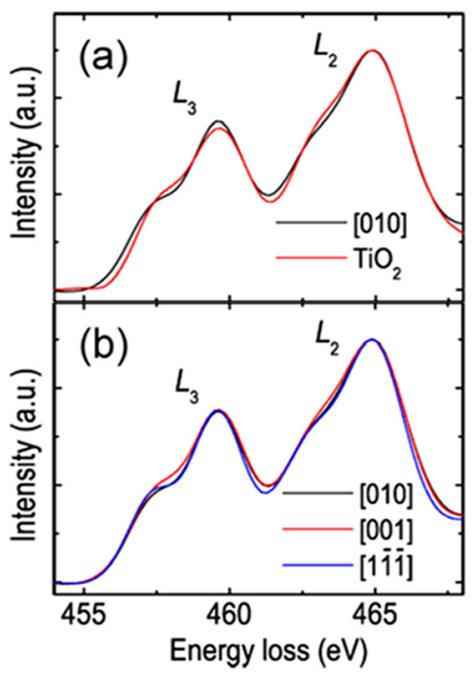

FIG. 4. (a) Comparison of the absorption edges of Ti- $L_{23}$ between BTF10 collected along [010] and $\mathrm{TiO}_{2}$. (b) The Ti- $L_{23}$ edges collected along different directions. Contribution of the plural scattering to the absorption edges is removed using the Fourier-log mode. edges of both BTF10 taken along the [010] direction and $\mathrm{TiO}_{2}$, the chemical shift clearly reveals the partial reduction of the ions from $\mathrm{Ti}^{4+}$ to $\mathrm{Ti}^{3+}$. In contrast, the absorption edges collected along different directions change slightly (see Fig. 4(b)). Hence, as observed in the ABF image, one out of two face-sharing octahedra transform into the edgesharing pyramids. This further verifies our analysis on the ABF image. Resembling to the formation of polaron ordering in $\operatorname{Pr}_{0.60} \mathrm{Ca}_{0.40} \mathrm{MnO}_{3}$, ${ }^{37}$ therefore, dynamic exchanges of trapped electrons among the polarons attribute to the unusual FM of BTF10. With respect to the interaction between the ordered polarons, dielectric properties of BTF10 provide us with more indications.

Figures 3(c) and 3(d) are the temperature dependence of $\varepsilon_{\mathrm{r}}$ and $\tan \delta$ around room temperature. In addition to the dielectric transitions taking place at $T_{\mathrm{C} 1}=430 \mathrm{~K}$ and $T_{\mathrm{C} 2}=345 \mathrm{~K}$, dielectric anomalies covering narrow temperature ranges appear around $T_{\mathrm{A} 1}=305 \mathrm{~K}$ as the frequency $(f)$ becomes larger than $20 \mathrm{kHz}$ and $T_{\mathrm{A} 2}=253 \mathrm{~K}$. For the anomaly around $T_{\mathrm{A} 2}, \varepsilon_{\mathrm{r}}$ shows a bump peak while $\tan \delta$ exhibits step-hopping behaviors with increasing temperature. In the frequency profile, branch-like $\tan \delta$ finally converges at $f=10 \mathrm{kHz}$ (see Fig. 3(e)). According to the electron paramagnetic resonance (EPR) experiments on ferroelectric $\mathrm{Pb}(\mathrm{Zr}, \mathrm{Ti}) \mathrm{O}_{3}$ and $\mathrm{BaTiO}_{3}{ }^{38}$ collective motion of Vos due to the alignment of interacting defect dipoles $\mathrm{Fe} 2^{3+}-\mathrm{Vö}$ and $\mathrm{Ti} 2^{3+}-\mathrm{Vö}$ (Vö: a doubly and positively charged Vo) may attribute to the abnormal dielectric behaviors. To figure out the essence of the dielectric anomalies and transitions, we performed the XRD experiments as a function of temperature. Our results reveal that no structural phase transition takes place within this temperature range (see Fig. 5). This further verifies that responses of the interacting defect dipoles to the ac $E$ attribute to the dielectric anomalies, while the dielectric transitions may possibly be caused by the accumulated motion of charged Vos, which finally leads to the structural phase transition under the excitation of $a c E$ and temperature.

Correspondingly, peculiar ferroelectric behavior of BTF10 reveals that it has improper FE at room temperature. Firstly, the polarization of the loops may experience a sudden enhancement as the applied $E$ surpasses a threshold field $\left(E_{\mathrm{th}}\right)$, e.g., $E_{\mathrm{th}}=15.9 \mathrm{kV} / \mathrm{cm}$ as $f=10 \mathrm{~Hz}$. Associated with the dielectric anomalies, a collective motion of charged Vos may

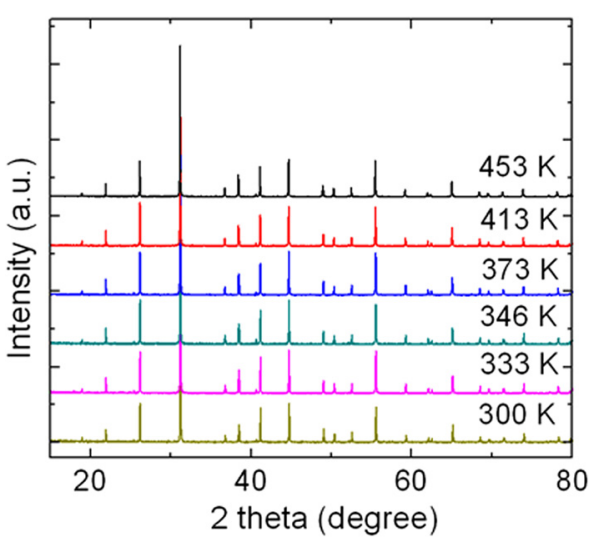

FIG. 5. The XRD pattern of BTF10 measured at higher temperatures. 
therefore explain the ferroelectric anomaly. Secondly, the leakage current plot shown in the insert of Fig. 3(f) not only reveals a highly insulating property $\left(\sim 10^{9} \Omega \mathrm{cm}\right)$ of the doped compound, but also a vacancy ordering induced asymmetric double potential well with a barrier height $E_{\mathrm{w}}=0.06 \mathrm{kV} / \mathrm{cm}$. Therefore, in spite of the $P-E$ loops not showing any typical ferroelectric character, they are essentially different from the cigar-shaped loops of lossy dielectrics. ${ }^{39}$

Supposing the edge-sharing pyramids dominate the polarization, polarization $P_{c a l}$ of the hexagonal matrix lying closely to $a, b$-plane can be approximately expressed as

$$
P_{c a l}=\frac{1}{V} \sum_{i}^{m} 2 n_{i} P_{0} \sin (\varphi / 2) \cos (\phi / 2),
$$

wherein, $V$ is the volume of a crystal, $n_{\mathrm{i}}$ is the number of unit cells for one polar orientation among three as indicated by $\phi=2 \pi / 3$, and $P_{0}$ is the dipole unit for one pyramid. This expression clearly reveals that the rotation angle $\phi$ and dihedral angle $\varphi$ are the primary order parameters of the improper ferroelectrics. ${ }^{40}$ Assuming $n_{\mathrm{i}}=m / 3(m$, the total unit cells), according to the parameters obtained from refinement, the estimated polarization value $P_{\text {cal }} \approx 2.1 \mu \mathrm{C} / \mathrm{cm}^{2}$ for a single crystal is about 10 times of that in the polycrystalline. Deviation of the refined structure from the true structure as well as grain boundaries in the doped sample attribute mainly to the polarization difference.

\section{Magnetoelectric effects}

With respect to the dynamic origin of FM, direct $E$ control of $M$ provides further verifications. Figure 6(a) shows the $M$ changes as a function of time, compared with the $M$ without applying $E$, and successive jumping of $M$ is observed as frequency increases from $f=100 \mathrm{~Hz}$ to $100 \mathrm{kHz}$. This reveals that the magnetic exchange interaction can be enhanced by increasing the hopping probabilities of the trapped electrons in the polarons. The frequency profile shown in the insert suggests that $E$ induced ME coupling coefficient has a logarithmic dependence on frequency. Alternatively, the ME coupling can also be driven by an applied $a c H$. As Fig. 6(b) shows, corresponding to the dielectric anomalies, the magnetically induced dynamic coupling coefficient $\alpha_{\mathrm{EM}}$ also show abnormal behaviors around $T_{\mathrm{A} 1}$ and $T_{\mathrm{A} 2}$. In addition to the gradual increase of $\alpha_{\mathrm{EM}}$ with increasing temperature, the isothermal $\alpha_{\mathrm{EM}}$ as a function of biased $d c H$ reveals voltage reversals between high and low states. From Figs. 6(c)-6(e), we see that the onset magnetic field $H_{\text {on }}$ decreases greatly with increasing temperature, e.g., from $H_{\text {on }}=11.5 \mathrm{kOe}$ at 243 $\mathrm{K}$ to $6.5 \mathrm{kOe}$ at $264 \mathrm{~K}$ then to $2.5 \mathrm{kOe}$ at $303 \mathrm{~K}$. Furthermore, as Fig. 6(f) shows, the coefficient $\alpha_{\mathrm{EM}}$ also logarithmically depends on frequency. These results verify that responses of trapped electrons in the polarons to $a c$ electric and magnetic fields attribute to the dynamically cross-controlled ME effect at room temperature.

\section{Discussion}

As for the origin mechanism of FM in this doped system, our previous studies and analysis on both dense $(\mathrm{x}=1 / 6,1 / 3)($ Ref. 18) and dilute $(\mathrm{x}=0.05)($ Ref. 16) Fe-doped samples have concluded that the density ratio between trapped electrons and mobile carriers fundamentally determines the ferromagnetic exchange interaction in the doped system. ${ }^{18}$ Comparison of the structural features between BTF10 and densely $(\mathrm{x}=1 / 6,1 / 3)$ doped samples may lead us to know more about the tunability of the physical properties of the doped system through controlling the variation of oxygen vacancy. (i) The Vos are orderly distributed at the $\mathrm{O} 1$ site only in the former, but randomly distributed at both $\mathrm{O} 1$ and $\mathrm{O} 2$ sites in the latter. Consequently, associated with the coordination changes of transition metals, the former is orderly distorted in the crystal structure, while the latter is incommensurately
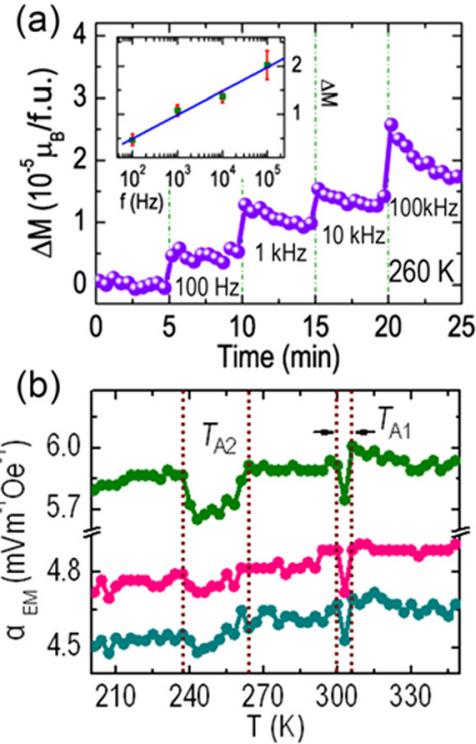
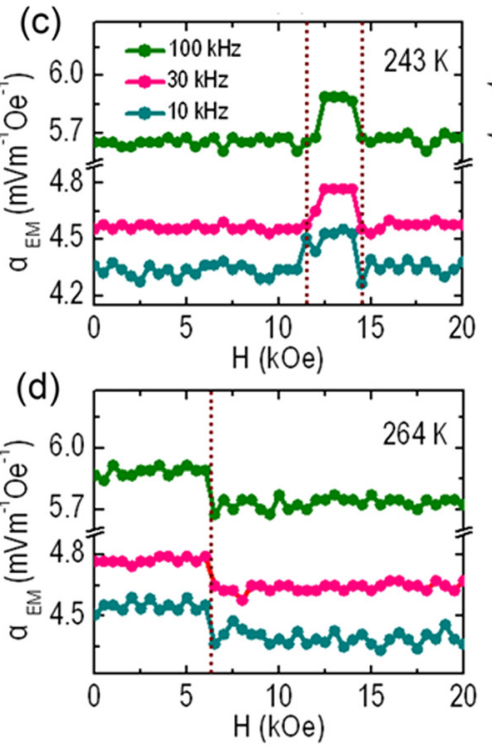
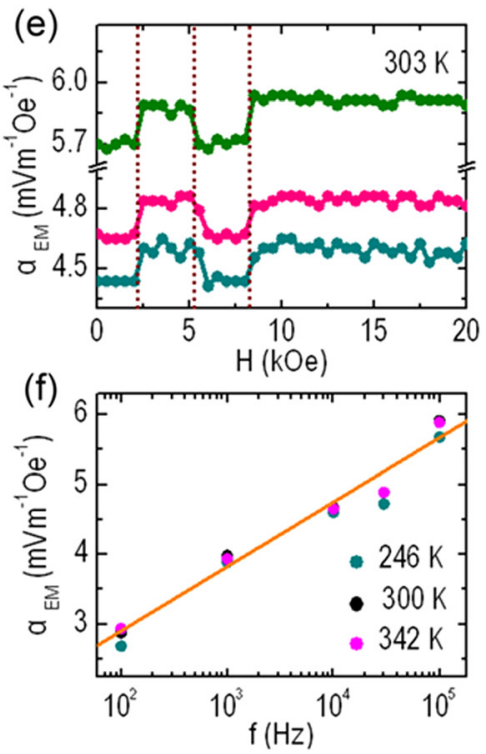

FIG. 6. (a) Time and frequency (the insert) profiles of $\Delta M$ induced by an $a c E$ at $260 \mathrm{~K}$. (b) Temperature dependence of magnetically induced $\alpha_{E M}$. (c)-(e) The $d c H$ dependence of $\alpha_{E M}$ measured at 243, 264, and $303 \mathrm{~K}$, respectively. (f) The frequency dependence of $\alpha_{E M}$ measured at different temperatures. 
modulated owing to the vacancy induced defect structures. (ii) The less doped Fe ions can be well accommodated at the M2 site in BTF10, but occupation of the dopants in the densely doped samples takes place at both M1 and M2 sites. Therefore, their differences in current-leaking, dielectric, and ferroelectric properties clearly suggest a coupling between the degree of structural order and the ability of charge bounding, which is also reflected from their differences in the magnetic property.

For the susceptibility data fitting in BTF10, the expression terminated at $p_{3}(H) \cdot T$ can also give a good fitting, but the best fitting is obtained from expression (1), which clearly reveals that $\chi^{\prime}=p_{1}(H) \cdot \mathrm{T}^{-1}$ attributes to the $\mathrm{PM}$ at $T<T_{\mathrm{P}-\mathrm{F}}$ while $\chi "=p_{2}(H)+p_{3}(H) \cdot T+p_{4}(H) \cdot T^{2}$ accounts for the ferromagnetic behavior at $T>T_{\mathrm{P}-\mathrm{F}}$. From the fitted parameters listed in Table I, the linear $p_{3}(H) \cdot T$ and the quadratic $p_{4}(H) \cdot T^{2}$ dominate the ferromagnetic exchange interaction especially at the high temperature region in BTF10. However, the quadratic term is detrimental to the explanation of magnetism origin in densely doped samples. ${ }^{18}$ This is mainly attributed to the influence of thermal fluctuation to the competition between the trapped electrons and the mobile carriers.

According to the dependencies of $\chi$ on $T, H$, and $f$, if the dynamic exchange interaction takes place only among limited trapped electrons within the nearest neighbors, it is hard to envisage the increase of $H_{\mathrm{C}}$ with the increase of temperature. On the contrary, if the exchange interaction takes place among more nearest-neighbor and next-nearest-neighbor trapped electrons, which are activated both by temperature and frequency, enhancement of the FM and $H_{\mathrm{C}}$ thus become reasonable and ferromagnetic domain growth can be envisaged. This is consistent with our magnetic and ME measurements. In addition, the charge transfer between the hybridized Ti-O bonding ${ }^{41}$ may possibly contribute slightly to the density of the trapped electrons, which could also favor the enhancement of the electric polarization by means of aligning the defect dipoles. ${ }^{38}$

As a matter of fact, the intricate coupling of these physical variants is fundamentally induced by the vacancy ordering of oxygen. First, dielectric anomalies and dielectric transitions, which are thoroughly different from that in the hexagonal $\mathrm{BaTiO}_{3},{ }^{42}$ are presented. Second, breaking of space inversion symmetry finally results in the improper FE at room temperature. Third, but not the last the dynamically cross-controlled ME effects are revealed at room temperature. With respect to the reversal of the magnetically induced voltage, similar to the ferroelectric anomaly, this may also attribute to the motion of charged Vos since the measurements are performed after the sample is poled. The rationality of this conclusion is further supported by the fact that this doped system has mixed (ionic and electronic) conductivity owing to the response of charged Vos to applied $E^{14,43}$ When compared with the reported results, ${ }^{10,11,16}$ our experiments point out that appearance of sufficient point defects, i.e., the vacancy content of oxygen, is a necessary condition for the generation of room-temperature FM in hexagonal $\mathrm{Ba}\left(\mathrm{Ti}_{1-\mathrm{x}} \mathrm{Fe}_{\mathrm{x}}\right) \mathrm{O}_{3-\delta}$ without the presence of $\mathrm{Fe}^{4+}$ ions. ${ }^{13}$ However, more fascinating physical properties can be revealed if an ordered point defect such as oxygen-vacancy ordering is introduced into the centrosymmetric matrix.

\section{CONCLUSIONS}

In summary, we carried out a detailed investigation on the structure, magnetic and electrical properties of $10 \mathrm{~mol} \%$ $\mathrm{Fe}$ doped hexagonal BaTiO3. Our experimental results point out that owing to the oxygen vacancy ordering, FM and improper FE become compatible at room temperature and responses of trapped electrons in the ordered polarons to magnetic and electric fields result in the cross-controlled ME coupling effects. By means of susceptibility data fitting and the ME measurements, the FM is concluded to derive from a dynamic phase transition from ground-state PM, i.e., the dynamic exchanges of trapped electrons in the polarons. In spite of the magnetoelectric coupling not being strong enough, the high ME coupling temperature, superior electrical insulating property, low manipulation fields, and cross-controlled performance may enable the new mechanism to be promising in designing novel multiferroic materials.

\section{ACKNOWLEDGMENTS}

This work was supported by the State Key Development Program for Basic Research of China (Grant No. 2012CB932302), the National Natural Science Foundation of China (Grant Nos. 11174336 and 50921091) and the specific funding of Discipline and Graduate Education Project of Beijing Municipal Commission of Education. L. Gu. is grateful for the support from the "Hundred Talents" program of the Chinese Academy of Sciences.

${ }^{1}$ W. Eerenstein, N. D. Mathur, and J. F. Scott, Nature 442, 759 (2006).

${ }^{2}$ T. Kimura, Y. Sekio, H. Nakamura, T. Siegrist, and A. P. Ramirez, Nature Mater. 7, 291 (2008).

${ }^{3}$ C.-Y. Ren, Phys. Rev. B 79, 125113 (2009).

${ }^{4}$ D. Higashiyama, S. Miyasaka, N. Kida, T. Arima, and Y. Tokura, Phys. Rev. B 70, 174405 (2004).

${ }^{5}$ A. Filippetti and N. Hill, Phys. Rev. B 65, 195120 (2002).

${ }^{6}$ D. V. Efremov, J. van den Brink, and D. I. Khomskii, Nature Mater. 3, 853 (2004).

${ }^{7}$ B. Xu, K. Yin, J. Lin, Y. Xia, X. Wan, J. Yin, X. Bai, J. Du, and Z. Liu, Phys. Rev. B 79, 134109 (2009).

${ }^{8}$ A. A. Belik, T. Furubayashi, Y. Matsushita, M. Tanaka, S. Hishita, and E. Takayama- Muromachi, Angew. Chem., Int. Ed. 48, 6117 (2009).

${ }^{9}$ K. Chen, F. Gao, W. W. Lin, H. L. Cai, G. L. Li, X. W. Dong, S. Peng, X. S. Wu, M. Yang, J. Du, X. M. Lu, J. M. Liu, and J. S. Zhu, Europhys. Lett. 89, 27004 (2010).

${ }^{10}$ S. Ray, P. Mahadevan, S. Mandal, S. Krishnakumar, C. Kuroda, T. Sasaki, T. Taniyama, and M. Itoh, Phys. Rev. B 77, 104416 (2008).

${ }^{11}$ F. Lin, D. Jiang, X. Ma, and W. Shi, J. Magn. Magn. Mater. 320, 691 (2008).

${ }^{12}$ F. Lin and W. Shi, J. Alloys Compd. 475, 64 (2009).

${ }^{13}$ F. Lin, D. Jiang, X. Ma, and W. Shi, Physica B 403, 2525 (2008).

${ }^{14}$ E. Mashkina, C. McCammon, and F. Seifert, J. Solid State Chem. 177, 262 (2004).

${ }^{15}$ I. E. Grey, C. Li, L. M. D. Cranswick, R. S. Roth, and T. A. Vanderah, J. Solid State Chem. 135, 312 (1998).

${ }^{16}$ T. Chakraborty, S. Ray, and M. Itoh, Phys. Rev. B 83, 144407 (2011).

${ }^{17}$ I. E. Grey, L. M. D. Cranswick, and C. Li, J. Appl. Crystallogr. 31, 692 (1998).

${ }^{18}$ X. K. Wei, Y. T. Su, Y. Sui, Q. H. Zhang, Y. Yao, C. Q. Jin, and R. C. Yu, J. Appl. Phys. 110, 114112 (2011).

${ }^{19}$ T. Kolodiazhnyi, A. A. Belik, S. C. Wimbush, and H. Haneda, Phys. Rev. B 77, 075103 (2008).

${ }^{20}$ D. C. Sinclair, J. M. S. Skakle, F. D. Morrison, R. I. Smith, and T. P. Beales, J. Mater. Chem. 9, 1327 (1999). 
${ }^{21}$ Y. Akishige, Y. Yamazaki, and N. Môri, J. Phys. Soc. Jpn. 73, 1267 (2004).

${ }^{22}$ G. M. Keith, M. J. Rampling, K. Sarma, N. M. Alford, and D. C. Sinclair, J. Eur. Ceram. Soc. 24, 1721 (2004).

${ }^{23}$ N. A. Hill, J. Phys. Chem. B 104, 6694 (2000).

${ }^{24}$ X. K. Wei, Q. H. Zhang, F. Y. Li, C. Q. Jin, and R. C. Yu, J. Alloys Compd. 508, 486 (2010).

${ }^{25}$ T. Zou, F. Wang, Y. Liu, L. Q. Yan, and Y. Sun, Appl. Phys. Lett. 97, 092501 (2010).

${ }^{26}$ H. M. Nguyen, N. V. Dang, P. Y. Chuang, T. D. Thanh, C. W. Hu, T. Y. Chen, V. D. Lam, C. H. Lee, and L. V. Hong, Appl. Phys. Lett. 99, 202501 (2011).

${ }^{27}$ N. Masó, H. Beltrán, E. Cordoncillo, P. Escribano, and A. R. West, J. Mater. Chem. 16, 1626 (2006).

${ }^{28}$ S. D. Findlay, N. Shibata, H. Sawada, E. Okunishi, Y. Kondo, and Y. Ikuhara, Ultramicroscopy 110, 903 (2010).

${ }^{29}$ Y. Tokura and N. Nagaosa, Science 288, 462 (2000).

${ }^{30}$ C.-H. Li, F. Wang, Y. Liu, X.-Q. Zhang, Z.-H. Cheng, and Y. Sun, Phys. Rev. B 79, 172412 (2009).

${ }^{31}$ G. Catalan and J. F. Scott, Adv. Mater. 21, 2463 (2009).
${ }^{32}$ L.-P. Chen, Y.-B Ma, X.-F. Song, G.-J. Lian, Y. Zhang, and G.-C. Xiong, Chin. Phys. Lett. 25, 3381 (2008).

${ }^{33}$ R. L. Carlin, Magnetochemistry (Springer-Verlag, Berlin, 1986), Chap. 2.

${ }^{34}$ O. Kahn, J. Galy, Y. Journaux, J. Jaud, and I. Morgenstern-Badarau, J. Am. Chem. Soc. 104, 2165 (1982).

${ }^{35}$ J. Galy, J. Jaud, O. Kahn, and P. Tola, Inorg. Chim. Acta 36, 229 (1979).

${ }^{36}$ G. M. Zhao, V. Smolyaninova, W. Prellier, and H. Keller, Phys. Rev. Lett. 84, 6086 (2000).

${ }^{37}$ A. Daoud-Aladine, J. Rodríguez-Carvajal, L. Pinsard-Gaudart, M. FernándezDíaz, and A. Revcolevschi, Phys. Rev. Lett. 89, 097205 (2002).

${ }^{38}$ W. L. Warren, G. E. Pike, K. Vanheusden, D. Dimos, B. A. Tuttle, and J. Robertson, J. Appl. Phys. 79, 9250 (1996).

${ }^{39}$ J. F. Scott, J. Phys.-Condens. Matter 20, 021001 (2008).

${ }^{40}$ A. P. Levanyuk and D. G. Sannikov, Sov. Phys. Usp. 17, 199 (1974).

${ }^{41}$ R. E. Cohen, Nature 358, 136 (1992).

${ }^{42}$ G. O. Yukikuni Akishige, T. Yamaoto, and E. Sawaguchi, J. Phys. Soc. Jpn. 58, 930 (1989).

${ }^{43}$ E. Mashkina, Ph.D. dissertation, Naturwissenschaftlichen Fakultäten der Friedrich-Alexander-Universität, 2005. 